\title{
Examination of general nutritional habits of students of Niğde Ömer Halisdemir University, academy of pyhsical education and sports
}

\author{
Meryem ALTUN, İ. Ümran AKDAĞCIK \\ Niğde Ömer Halisdemir University Academy of Physical Education and Sports, Niğde, Turkey \\ Address correspondence to M, Altun, e-mail: mrymltn@hotmail.com
}

\begin{abstract}
The purpose of this study is to examine the general nutritional habits of students studying at Niğde Ömer Halisdemir University, School of Physical Education and Sports. Through voluntary participation -as being 89 males and 94 females- 180 students in total. General Nutrition Scale whose reliability has been realized by Öztürk (2017) had been used as data collection tool. In the analysis of data, percentage and frequency had been used for demographical features, and ANOVA had been used for descriptive statistics and for the determination of differences among groups. Consequently, while a significant difference had been determined in between the variable of age and the propositions regarding attitudes towards general nutrition, and in between the variables of gender, height and weight and the propositions regarding behaviors towards food supplements. In the propositions regarding behaviors towards food supplements, it had been determined that the averages are high in favor of males. While a significant difference had been determined in the variable of department and in propositions regarding attitudes towards general nutrition. In the propositions regarding attitudes towards general nutrition, it had been determined that it is in favor of the students of Department of Coaching from among the students of Department of Coaching and students of Department of Sports Management. While a significant relation in positive direction had been determined in between the variable of weekly attendance to gym and propositions regarding behaviors towards additive containing nutrition. It has been revealed that the individuals attending the gym exhibit a more positive attitude regarding the use of additive containing substances compared to the ones not attending the gym. While a significant relation in positive direction had been determined in between the variable of number of meals and propositions regarding behaviors towards additive containing nutrition, no significant relation had been found among the other propositions.
\end{abstract}

Keywords: Academy of Physical Education and Sports, Nutrition, Food supplements

\section{INTRODUCTION}

The concept of nutrient or nutritional element, which is known as things eaten and drank in the simplest form, expresses organic and inorganic chemical substances used for the organism to subsist and to meet its energy requirement, and for the formation and repair of tissues (4). In this case, there is no inconvenience in defining the nutrition behavior as the consumption of nutritional elements. On the other hand, it is required not to overlook that nutrition is a science as well as being an act. And nutrition as a branch of science concerns with researching the types of nutrients and nutritional elements, their features and duties, and the factors affecting their quality, and researching, preparing and applying suitable nutrition forms for the individuals to be nourished in a balanced and healthy manner (13).
Nutrition is an essential and significant factor in terms of human life. The activity of nutrition, being required in order to be healthy and in order to maintain vital functions, has became a problem required to be solved since the initial seconds of history of humanity. And the solution of this difficult position occurring in nutrition, which became a greater problem in terms of being solved depending on the increasing population today, has been additive containing nutrients. Even if the additive containing nutrients, which started to threat more the health of individuals and society each passing day, are frequently being consumed due to their inexpensiveness and extensiveness, it is still unknown how that inexpensiveness will be atoned. On the other hand, it can be said that food supplements, which became more popular especially due to changes in the perception of body sizes and form, are constituting another problematic area of nutrition manners of today. In this respect, it 
is clear that the attitudes and behaviors of today's societies and individuals against these two factors are very important (16).

The state of insufficient or unbalanced nutrition, which can be characterized as state in which sufficient and balance nutrition is not realized, basically indicates lack of intake of nutritional elements at required amounts or instability at the level of nutritional elements taken. This condition is inviting various health problems and many physiological and psychological illnesses. For instance, problems such as failure in growth and development, defects in structural and organic functions of the brain, lack of attention, and inadequacy in learning activities are able to be observed in undernourished individuals (2).

Genetic, physiological and psychological factors, health state, type of exercise and nutrition are the basic factors affecting the performance in athletes (8). Among these factors, as nutrition is directly affecting the health and performance of the athlete, it is a subject which should be pondered on. It is obligatory for the athletes to be nourished correctly in order to attain the high performance they require. An athlete, who cannot ensure her/his nutrition in a sufficient and balanced manner, will not be able to attain the efficiency and success s/he wants, and will have a weak immune system against infections, and will have muscles whose period of recovery will take longer in cases of injuries (9).

Based on this information, research of general nutrition habits of individuals, their attitudes and behavior towards additive containing nutrition, and their attitudes and behaviors towards food supplements is important in terms of providing sufficient information for the students of Academy of Physical Education and Sports regarding nutritional habits. Thus, general nutritional habits of students studying at Niğde Ömer Halisdemir University, School of Physical Education and Sports had been examined.

\section{MATERIAL \& METHOD}

\section{Model of Research}

This Screening model had been used in the research. Screening models is a research approach intending to portray a past or current status as is (6).

\section{Study Group}

Students of Academy of Physical Education and Sports areconstituting the universe of the research,

Turk J Sport Exe 2018; 20(3): I63 - 173

๑ 2018 Faculty of Spart Sciences, Selcuk University and students of Niğde Ömer Halisdemir University Academy of Physical Education and Sports are constituting the sample of the research. 180 students in total -as being 89 males and 94 females- studying at departments of Coaching Education ( $n=60)$, Physical Education and Sports Teaching $(n=60)$ and Sports Management $(n=60)$ had participated in the research.

\section{Data Collection Tool}

During the determination of propositions used in the questionnaire, scales -used in studies of Sezek et al. (2008)- had been used by making some changes, and also various new propositions relevant to food supplements had been added (18). And as a result, 40 questions in total -as 39 being Likert typehad been included in the scale. In the questionnaire, 17 of these Likert type propositions are relevant to "General Nutrition Attitudes", 7 of them are relevant to "Attitudes Towards Additive Containing Nutrition", 5 of them are relevant to "Attitudes Towards Food Supplements", 6 of them are relevant to "Behaviors Towards Additive Containing Nutrition", and 4 of them are relevant to "Behaviors Towards Food Supplements". When the Cronbach's Alpha Test result of the scale used in the study is considered, it has been understood that the model's general significance level is .734. If it is considered that for a questionnaire to be reliable it is required to have a reliability value of over .700 , it can be said that the questionnaire / scale used in the study is successful (16).

\section{Analysis of Data}

The data had been analyzed by SPSS 21 packaged software. In the analysis of data, percentage and frequency had been used for demographical features, and ANOVA had been used for descriptive statistics and for the determination of differences among groups. For the solution of sub-problems, correlation analysis (Pearson Correlation Moment) had been applied in order to examine the relation among the variables of participants' age, height, weight and period of attendance to gym, and Independent Sample t-test had been applied for the variable of gender. 


\section{INTERPRETATION OF ANALYSIS AND} FINDING

Table 1. Demographical features of the participants

\begin{tabular}{cccccc}
\hline & $\overline{\boldsymbol{x}}$ & Ss & Min. & Max. & Total \\
\hline Age & 21.80 & 1.80 & 18.00 & 28.00 & 180 \\
\hline Height & 171.89 & 8.93 & 150.00 & 190.00 & 180 \\
\hline Weight & 65.51 & 13.25 & 42.00 & 116.00 & 180 \\
\hline
\end{tabular}

Table 2. Frequency and percentage values regarding the variables of participants' gender and department

\begin{tabular}{clllc}
\hline & & f & \% & Total \\
\hline Gender & Male & 86 & 47.8 & \multirow{2}{*}{180} \\
\hline Depart & Female & 94 & 52.2 & \\
ment & Coaching Education & 60 & 33.3 & \\
& Physical Education & 60 & 33.3 & \multirow{2}{*}{180} \\
& Teaching & 60 & 33.3 & \\
& Sports Management & & & \\
\hline
\end{tabular}

Table 3. Arithmetic mean and standard deviation values regarding participants' period of attendance to gym

\begin{tabular}{cccccc}
\hline & $\overline{\boldsymbol{x}}$ & Ss & Min. & Max & Total \\
\hline $\begin{array}{c}\text { Daily period of } \\
\text { attendance to gym }\end{array}$ & 1,90 & 0,94 & 1 & 6 & 133 \\
\hline $\begin{array}{c}\text { Weekly period of } \\
\text { attendance to gym }\end{array}$ & 3,15 & 1,28 & 1 & 7 & 133 \\
\hline
\end{tabular}

Table 4. Frequency and percentage values regarding the variable of participants' reason of attending the gym

\begin{tabular}{ccccc}
\hline & & $\mathbf{f}$ & $\mathbf{\%}$ & Total \\
\hline & Body Building & 40 & 22,2 & \\
\cline { 2 - 4 } Reason of \\
attending & Losing Weight & 10 & 5,6 & \\
\cline { 2 - 4 } the gym & Gaining Weight & 3 & 1,7 & \multirow{2}{*}{134} \\
\cline { 2 - 4 } & Health & 29 & 16,1 & \\
\cline { 2 - 4 } & Condition & 30 & 16,7 & \\
\cline { 2 - 4 } & Keeping Fitness & 22 & 12,2 \\
\hline
\end{tabular}

Table 5. Frequency and percentage values regarding the participants' meal skipping status

\begin{tabular}{|c|c|c|c|c|}
\hline & & $\mathrm{f}$ & $\%$ & Total \\
\hline \multirow{5}{*}{$\begin{array}{c}\text { Skipping } \\
\text { meal }\end{array}$} & I don't skip & 45 & 25,0 & \multirow{5}{*}{180} \\
\hline & I do skip & 71 & 39,4 & \\
\hline & $\begin{array}{c}\text { I do skip, generally } \\
\text { the breakfast }\end{array}$ & 30 & 16,7 & \\
\hline & $\begin{array}{c}\text { I do skip, generally } \\
\text { lunch }\end{array}$ & 31 & 17,2 & \\
\hline & $\begin{array}{c}\text { I do skip, generally } \\
\text { dinner }\end{array}$ & 2 & 1,1 & \\
\hline
\end{tabular}

When the findings in Table 7 are examined, in Propositions Regarding Attitudes Towards Genera Nutrition, average response intensity for the proposition of "Breakfast is an important meal" is 4.72; and average response intensity for the proposition of "Refreshments are unnecessary" is
Table 6. Frequency and percentage values regarding the participants' meal skipping reason

\begin{tabular}{ccccc}
\hline & f & \% & Total \\
\hline & $\begin{array}{c}\text { Losing weight as } \\
\text { easting less }\end{array}$ & 7 & 3,9 & \\
\cline { 2 - 4 } & Lack of time & 68 & 37,8 & \\
\cline { 2 - 4 } $\begin{array}{c}\text { Reason of } \\
\text { skipping } \\
\text { meal }\end{array}$ & Economical reasons & 12 & 6,7 & \\
\cline { 2 - 4 } & Inappetence & 31 & 17,2 & \multirow{2}{*}{180} \\
\cline { 2 - 4 } & Choosy eating & 6 & 3,3 & \\
\cline { 2 - 4 } & Other & 6 & 3,3 \\
\cline { 2 - 4 } & Keeping fitness & 2 & 1,1 \\
\cline { 2 - 3 } & Not liking to eat out & 2 & 1,1 \\
\hline
\end{tabular}

2.21. In the Propositions Regarding Attitudes Towards Additive Containing Nutrition, average response intensity for the proposition of "By the additive containing nutrients, problems of famine and hunger may be solved in the country and in the world." is 2.59; average response intensity for the proposition of "Use of food supplements causing to lose weight is not harmful for losing weight." is 2.18. In the Propositions Regarding Attitudes Towards Food Supplements, average response intensity for the proposition of "The ones doing exercise should definitely use food supplements." is 3.16; average response intensity for the proposition of "Use of food supplements causing to lose weight is not harmful for losing weight." is 2.18. In Propositions Regarding Behaviors Towards Additive Containing Nutrition, average response intensity for the proposition of "During shopping, I examine the product labels, and try to understand the content of nutrient." is 3.59; average response intensity for the proposition of "I just consume natural products." is 3.03. In Proposition Regarding Behaviors Towards Food Supplements, average response intensity for the proposition of "I use food supplements on a daily basis." is 2.74; average response intensity for the proposition of "I use Le-carnetin etc. products for accelerating burning of fat." is 2.16 . 
Table 7. Participants' average density regarding AGN. AACN. AFS. BACN and BFS

\begin{tabular}{|c|c|}
\hline Factors & Average \\
\hline \multicolumn{2}{|l|}{ Propositions Regarding Attitudes Towards General Nutrition } \\
\hline Breakfast is an important meal. & 4.72 \\
\hline At least 3 meals should be had per day. & 4.38 \\
\hline Refreshments are unnecessary. & 2.11 \\
\hline It is required to consider the daily tea-coffee consumption, and not to exceed specific limits. & 3,94 \\
\hline I believe eating a little and frequently is beneficial. & 3,97 \\
\hline At least 2 liters water should be consumer per day, and it should even me more in case of doing exercise. & 4,60 \\
\hline I think frying is an unhealthy method. & 2.80 \\
\hline Excessive salt and sugar use negatively affects health. & 4.03 \\
\hline The ones having high cholesterol should prefer vegetal food. & 3.73 \\
\hline It is required to use vegetable oils instead of butter. & 3.04 \\
\hline Use of full-fat yoghurt and milk by adults is better than fat free products. & 3.34 \\
\hline Oil shouldn't be used while cooking meat. & 3.02 \\
\hline Consuming fish once a month is sufficient. & 2.89 \\
\hline The ones doing exercise should definitely consume meat. & 4.34 \\
\hline While eating meat such as chicken. turkey etc.. their skin should definitely be removed. & 3.76 \\
\hline It is required to frequently consume rice without making distinction of cracked wheat or rice. & 3.32 \\
\hline During shopping. the labels of products should be examined. and information should be had about nutrient content. & 4.21 \\
\hline \multicolumn{2}{|l|}{ Propositions Regarding Attitudes Towards Additive Containing Nutrition } \\
\hline Additive containing nutrients are no different than the natural products. & 1.56 \\
\hline Additives are beneficial due to extending the shelf life and service life of natural products. & 2.03 \\
\hline $\begin{array}{l}\text { Additive containing nutrients are products with higher nutritional value compared to natural products due to the } \\
\text { additives they include. }\end{array}$ & 2.22 \\
\hline $\begin{array}{l}\text { Additives ensure the natural products to be relieved from problems due to hygienic mistakes. harmful content and } \\
\text { incorrect disinfection. }\end{array}$ & 2.36 \\
\hline By the additive containing nutrients, problems of famine and hunger may be solved in the country and in the world. & 2,59 \\
\hline As the additives are increasing the taste of natural products, they are making eating more enjoyable and easy. & 2,55 \\
\hline Additive containing nutrients are not unhealthy. & 2,19 \\
\hline \multicolumn{2}{|l|}{ Propositions Regarding Attitudes Towards Food Supplements } \\
\hline Food supplements are not harmful. & 2,98 \\
\hline The ones doing exercise should definitely use food supplements. & 3,16 \\
\hline Protein powder etc. products are definitely required to be used for body building. & 2,48 \\
\hline Use of food supplements causing to lose weight is not harmful for losing weight. & 2.18 \\
\hline The ones doing exercise should definitely consume energy drinks. & 2.67 \\
\hline \multicolumn{2}{|l|}{ Propositions Regarding Behaviors Towards Additive Containing Nutrition } \\
\hline$I^{\prime} m$ careful about additive containing nutrients in my daily nutrition. & 3.35 \\
\hline I care not to consume nutrients that I don't know about their content. & 3.51 \\
\hline During shopping. I examine the product labels. and try to understand the content of nutrient. & 3.59 \\
\hline I just consume natural products. & 3.03 \\
\hline I consume from organic product sections. & 3.41 \\
\hline I try to make the foodstuff such as sausage, yoghurt, tomato paste, pickle, canned food etc. at home by myself. & 3.33 \\
\hline \multicolumn{2}{|l|}{ Propositions Regarding Behaviors Towards Food Supplements } \\
\hline I use food supplements on a daily basis. & 2.74 \\
\hline I use protein powder in order to accelerate muscle development while doing exercise. & 2.26 \\
\hline I use Le-carnetin etc. products for accelerating burning of fat. & 2.16 \\
\hline I frequently drink energy drinks in order to meet my energy requirement. & 2.23 \\
\hline
\end{tabular}

Table 8. Values of relation in between participants' age variable and AGN, AACN, AFS, BACN and BFS propositions

\begin{tabular}{cccc}
\hline & Age & & p \\
\hline & $\mathbf{N}$ & $\mathbf{r}$ & 0.05 \\
\hline General Nutrition Attitudes & 180 & $0.15^{*}$ & 0.68 \\
\hline Attitudes Towards Additive Containing Nutrition & 180 & -0.03 & 0.20 \\
\hline Attitudes Towards Food Supplements & 180 & 0.10 & 0.08 \\
\hline Behaviors Towards Additive Containing Nutrition & 180 & 0.13 & 0.09 \\
\hline Behaviors Towards Food Supplements & 180 & 0.13 & \\
\hline
\end{tabular}

When Table 8 is examined, while a significant $(\mathrm{r}=0.15 ; \mathrm{p}=0.05)$, no significant relation had been relation in positive direction had been determined in found among other propositions. between participants' age variable and Propositions Regarding Attitudes Towards General Nutrition 
Table 9. T-test results of participants' gender and AGN, AACN, AFS, BACN and BFS propositions

\begin{tabular}{|c|c|c|c|c|c|c|c|}
\hline & Gender & $\mathbf{n}$ & $\overline{\bar{x}}$ & $S$ & sd & $t$ & $p$ \\
\hline \multirow{2}{*}{ General Nutrition Attitudes } & Male & 86 & 3.69 & 0.54 & \multirow{2}{*}{178} & \multirow{2}{*}{0.89} & \multirow{2}{*}{0.37} \\
\hline & Female & 94 & 3.63 & 0.40 & & & \\
\hline \multirow{2}{*}{ Attitudes Towards Additive Containing Nutrition } & Male & 86 & 2.22 & 0.83 & \multirow{2}{*}{178} & \multirow{2}{*}{0.07} & \multirow{2}{*}{0.94} \\
\hline & Female & 94 & 2.21 & 0.93 & & & \\
\hline \multirow{2}{*}{ Attitudes Towards Food Supplements } & Male & 86 & 3.49 & 0.77 & \multirow{2}{*}{178} & \multirow{2}{*}{0.95} & \multirow{2}{*}{0.35} \\
\hline & Female & 94 & 3.39 & 0.66 & & & \\
\hline \multirow{2}{*}{ Behaviors Towards Additive Containing Nutrition } & Male & 86 & 3.35 & 0.79 & \multirow{2}{*}{178} & \multirow{2}{*}{-036} & \multirow{2}{*}{0.72} \\
\hline & Female & 94 & 3.39 & 0.76 & & & \\
\hline \multirow{2}{*}{ Behaviors Towards Food Supplements } & Male & 86 & 2.51 & 1.00 & \multirow{2}{*}{178} & \multirow{2}{*}{2.09} & \multirow{2}{*}{0.04} \\
\hline & Female & 94 & 2.19 & 1.03 & & & \\
\hline
\end{tabular}

When the findings in Table 9 are examined, while a significant difference had been determined in between the participants' gender and Propositions Regarding Behaviors Towards Food Supplements $(t=2.09 ; \quad p=0.04)$, no significant difference had been found among other

When arithmetic means are reviewed for the analysis of significant difference, it had been determined that the averages were high in favor of males in Propositions Regarding Behaviors Towards Food Supplements (Male =2.51; Female =2.19). propositions.

Table 10. Values of relation in between participants' height variable and AGN, AACN, AFS, BACN and BFS propositions

\begin{tabular}{cccc}
\hline & & Height & $\mathbf{p}$ \\
\hline General Nutrition Attitudes & $\mathbf{n}$ & $\mathbf{r}$ & 0.25 \\
\hline Attitudes Towards Additive Containing Nutrition & 180 & 0.09 & 0.42 \\
\hline Attitudes Towards Food Supplements & 180 & 0.06 & 0.52 \\
\hline Behaviors Towards Additive Containing Nutrition & 180 & 0.05 & 0.38 \\
\hline Behaviors Towards Food Supplements & 180 & 0.07 & 0.02 \\
\hline
\end{tabular}

When Table 10 is examined, while a significant Propositions Regarding Behaviors Towards relation in positive direction had been determined in Food Supplements $(\mathrm{r}=0.17$; $\mathrm{p}=0.02)$, no significant between participants' height variable and

relation had been found among other propositions.

Table 11. Values of relation in between participants' weight variable and AGN. AACN. AFS. BACN and BFS propositions

\begin{tabular}{|c|c|c|c|}
\hline & \multicolumn{3}{|c|}{ Weight } \\
\hline & $\mathbf{n}$ & $\mathbf{r}$ & p \\
\hline General Nutrition Attitudes & 180 & -0.01 & 0.90 \\
\hline Attitudes Towards Additive Containing Nutrition & 180 & 0.06 & 0.45 \\
\hline Attitudes Towards Food Supplements & 180 & -0.01 & 0.90 \\
\hline Behaviors Towards Additive Containing Nutrition & 180 & -0.01 & 0.87 \\
\hline Behaviors Towards Food Supplements & 180 & $0.24^{* *}$ & 0.00 \\
\hline
\end{tabular}

The value When Table 11 is examined, while a significant relation in positive direction had been determined in between participants' weight variable and Propositions Regarding Behaviors Towards Food Supplements ( $\mathrm{r}=0.24 ; \mathrm{p}=0.00)$, no significant relation had been found among other propositions.

When the findings in Table 12 are examined, while significant difference had been determined in participants' department variable and in Propositions Regarding Attitudes Towards Genera Nutrition $(F(2,177)=3,18 ; \quad p<0,05)$, no significant difference had been found in other propositions.
When the Post Hoc Tukey findings and arithmetic means are compared, it had been determined that the significant difference is in favor of students of Department of Coaching -from among the students of Department of Coaching, and of Department of Sports Management- in Propositions Regarding Attitudes Towards General Nutrition. 
Table 12. Results of one-way analysis of variance (anova) in between participants' department variable and AGN, AACN, AFS, BACN and BFS

\begin{tabular}{|c|c|c|c|c|c|c|c|c|c|c|}
\hline & Group & $\mathbf{N}$ & $\overline{\boldsymbol{x}}$ & ss & Var. Coeff. & K. T & sd & K. O & F & $\mathrm{p}$ \\
\hline \multirow{5}{*}{ General Nutrition Attitudes } & Coaching & 60 & 3,76 & 0.37 & Among & 1.371 & 2 & 0.685 & \multirow{3}{*}{3.18} & \multirow{3}{*}{0.04} \\
\hline & & & & & Groups & & & & & \\
\hline & PE Teaching & 60 & 3.66 & 0.48 & Within Group & 38.189 & 177 & 0.216 & & \\
\hline & Sports Management & 60 & 3.55 & 0.53 & Total & 39.560 & 179 & & & \\
\hline & Total & 180 & 366 & 0.47 & $\begin{array}{l}\text { Among } \\
\text { Groups }\end{array}$ & & & & & \\
\hline \multirow{4}{*}{$\begin{array}{l}\text { Attitudes Towards Additive Containing } \\
\text { Nutrition }\end{array}$} & Coaching & 60 & 2.29 & 0.98 & Within Group & 2.899 & 2 & 1.449 & \multirow{2}{*}{1.89} & \multirow{2}{*}{0.16} \\
\hline & PE Teaching & 60 & 2.32 & 0.90 & Total & 135.938 & 177 & 0.768 & & \\
\hline & Sports Management & 60 & 2.04 & 0.74 & $\begin{array}{l}\text { Among } \\
\text { Groups }\end{array}$ & 138.837 & 179 & & & \\
\hline & Total & 180 & 2,21 & 0,88 & Within Group & & & & & \\
\hline \multirow{4}{*}{ Attitudes Towards Food Supplements } & Coaching & 60 & 3,57 & 0,66 & Total & 2,736 & 2 & 1,368 & \multirow[b]{2}{*}{2,73} & \multirow[b]{2}{*}{0,07} \\
\hline & PE Teaching & 60 & 3,45 & 0,73 & $\begin{array}{l}\text { Among } \\
\text { Groups }\end{array}$ & 88,664 & 177 & 0,501 & & \\
\hline & Sports Management & 60 & 3,27 & 0,74 & Within Group & 91,400 & 179 & & & \\
\hline & Total & 180 & 3,43 & 0,71 & Total & & & & & \\
\hline \multirow{5}{*}{$\begin{array}{c}\text { Behaviors Towards Additive Containing } \\
\text { Nutrition }\end{array}$} & Coaching & 60 & 3.40 & 0.67 & Among & 0.523 & 2 & 0.261 & \multirow{3}{*}{0.44} & \multirow{3}{*}{0.65} \\
\hline & & & & & Groups & & & & & \\
\hline & PE Teaching & 60 & 3.41 & 0.83 & Within Group & 106.231 & 177 & 0.600 & & \\
\hline & Sports Management & 60 & 3.29 & 0.81 & Total & 106.753 & 179 & & & \\
\hline & Total & 180 & 3.70 & 0.77 & $\begin{array}{l}\text { Among } \\
\text { Groups }\end{array}$ & & & & & \\
\hline & Coaching & & & & Within Group & 5.527 & 2 & 2.764 & & \multirow{2}{*}{0.07} \\
\hline \multirow{4}{*}{ Behaviors Towards Food Supplements } & PE Teaching & 60 & 2.56 & 1.00 & Total & 182.757 & 177 & 1.033 & & \\
\hline & Sports Management & 60 & 2.34 & 1.05 & $\begin{array}{l}\text { Among } \\
\text { Groups }\end{array}$ & 188.284 & 179 & & & \\
\hline & Total & 60 & 2.13 & 1.00 & Within Group & 1.371 & 2 & 0.685 & 3.18 & 0.04 \\
\hline & & 180 & 2.35 & 1.03 & Total & & & & & \\
\hline & & \multicolumn{2}{|c|}{ Section (A) } & \multicolumn{2}{|c|}{ Section (B) } & \multicolumn{2}{|c|}{$\begin{array}{l}\text { Mean Dif. } \\
\text { (A-B) }\end{array}$} & \multicolumn{2}{|l|}{ Std. Er. } & $\mathrm{p}$ \\
\hline \multicolumn{2}{|c|}{ General Nutrition Attitudes } & \multicolumn{2}{|c|}{ Coaching } & \multicolumn{2}{|c|}{ Sports Management } & \multicolumn{2}{|c|}{$0.21^{*}$} & \multicolumn{2}{|l|}{0.08} & 0.03 \\
\hline
\end{tabular}

Table 13. Values of relation in between participants' period of daily attendance to gym variable and AGN. AACN. AFS. BACN and BFS propositions

\begin{tabular}{lccc}
\hline & \multicolumn{3}{c}{$\begin{array}{c}\text { Period of Daily } \\
\text { Attendance to Gym }\end{array}$} \\
\hline & $\mathbf{n}$ & $\mathbf{r}$ & $\mathbf{p}$ \\
\hline General Nutrition Attitudes & 133 & 0.11 & 0.22 \\
\hline $\begin{array}{l}\text { Attitudes Towards Additive } \\
\text { Containing Nutrition }\end{array}$ & 133 & 0.14 & 0.11 \\
\hline $\begin{array}{l}\text { Attitudes Towards Food } \\
\text { Supplements }\end{array}$ & 133 & -0.04 & 0.62 \\
\hline $\begin{array}{l}\text { Behaviors Towards Additive } \\
\text { Containing Nutrition }\end{array}$ & 133 & -0.04 & 0.63 \\
\hline $\begin{array}{l}\text { Behaviors Towards Food } \\
\text { Supplements }\end{array}$ & 133 & 0.10 & 0.25 \\
\hline
\end{tabular}

When Table 13 is examined. no significant relation had been found among the participants' period of daily attendance to gym variable and Propositions Regarding Attitudes Towards General Nutrition, Proposition Regarding Attitudes Towards Additive Containing Nutrition, Propositions Regarding Attitudes Towards Food Supplements, Propositions Regarding Behavior Towards Additive Turk ل Sport Exe 2018; 20(3): 163 - 173 ๑ 2018 Faculty of Sport Sciences, Selcuk University
Containing Nutrition and Propositions Regarding Behaviors Towards Additive Containing Nutrition.

Table 14. Values of relation in between participants' period of weekly attendance to gym variable and AGN, AACN, AFS, BACN and BFS propositions

\begin{tabular}{lccc}
\hline & \multicolumn{3}{c}{$\begin{array}{c}\text { Period of Weekly } \\
\text { Attendance to Gym }\end{array}$} \\
\hline & $\mathbf{n}$ & $\mathbf{r}$ & $\mathbf{p}$ \\
\hline General Nutrition Attitudes & 133 & -0.03 & 0.73 \\
\hline $\begin{array}{l}\text { Attitudes Towards Additive } \\
\text { Containing Nutrition }\end{array}$ & 133 & -0.08 & 0.36 \\
\hline Attitudes Towards Food Supplements & 133 & -0.08 & 0.36 \\
\hline $\begin{array}{l}\text { Behaviors Towards Additive } \\
\text { Containing Nutrition }\end{array}$ & 133 & $0.21^{*}$ & 0.01 \\
\hline Behaviors Towards Food Supplements & 133 & 0.01 & 0.91
\end{tabular}

When Table 14 is examined, while a significant relation in positive direction had been determined in between participants' period of weekly attendance to gym variable and Propositions Regarding Behaviors Towards Additive Containing Nutrition $(\mathrm{r}=0.21 ; \mathrm{p}=0.01)$, no significant relation had been found among other propositions. 
Table 15. Results of one-way analysis of variance (anova) in between participants' reason of attendance to gym variable and AGN, AACN, AFS, BACN and BFS

\begin{tabular}{|c|c|c|c|c|c|c|c|c|c|c|}
\hline & \multicolumn{2}{|l|}{ Group } & \multicolumn{2}{|l|}{$\overline{\bar{x}}$} & \multirow[t]{2}{*}{ Var. Coeff. } & \multirow[b]{2}{*}{ K. T } & \multirow[b]{2}{*}{ sd } & \multirow[b]{2}{*}{ K. O } & \multirow[b]{2}{*}{ F } & \multirow[b]{2}{*}{$\mathrm{p}$} \\
\hline & & $\mathbf{N}$ & & ss & & & & & & \\
\hline \multirow{8}{*}{ General Nutrition Attitudes } & Body Building & 40 & 3.71 & 0.60 & Among & 1.535 & 5 & 0.307 & \multirow{3}{*}{1.47} & \multirow{3}{*}{0.21} \\
\hline & & & & & Groups & & & & & \\
\hline & Losing Weight & 10 & 3.61 & 0.52 & Within Group & 26.822 & 128 & 0.210 & & \\
\hline & Gaining Weight & 3 & 3.51 & 0.22 & Total & 28.358 & 133 & & & \\
\hline & Health & 29 & 3.49 & 0.33 & & & & & & \\
\hline & Conditioning & 30 & 3.76 & 0.41 & & & & & & \\
\hline & Fitness & 22 & 3.76 & 0.34 & & & & & & \\
\hline & Total & 134 & 3.67 & 0.46 & & & & & & \\
\hline \multirow{8}{*}{$\begin{array}{l}\text { Attitudes Towards Additive Containing } \\
\text { Nutrition }\end{array}$} & Body Building & 40 & 2.47 & 0.87 & Among & 6.707 & 5 & 1.341 & \multirow{3}{*}{1.94} & \multirow{3}{*}{0.09} \\
\hline & & & & & Groups & & & & & \\
\hline & Losing Weight & 10 & 2.41 & 1.03 & Within Group & 88.626 & 128 & 0.692 & & \\
\hline & Gaining Weight & 3 & 1.86 & 1.36 & Total & 95.333 & 133 & & & \\
\hline & Health & 29 & 1.90 & 0.76 & & & & & & \\
\hline & Conditioning & 30 & 2.26 & 0.79 & & & & & & \\
\hline & Fitness & 22 & 2.01 & 0.75 & & & & & & \\
\hline & Total & 134 & 2.22 & 0.84 & & & & & & \\
\hline \multirow{8}{*}{ Attitudes Towards Food Supplements } & Body Building & 40 & 3.61 & 0.75 & Among & 4.352 & 5 & 0.870 & \multirow{3}{*}{1.72} & \multirow{3}{*}{0.14} \\
\hline & & & & & Groups & & & & & \\
\hline & Losing Weight & 10 & 3.54 & 0.91 & Within Group & 64.881 & 128 & 0.507 & & \\
\hline & Gaining Weight & 3 & 3.27 & 0.31 & Total & 69.232 & 133 & & & \\
\hline & Health & 29 & 3.15 & 0.69 & & & & & & \\
\hline & Conditioning & 30 & 3.53 & 0.68 & & & & & & \\
\hline & Fitness & 22 & 3,57 & 0,64 & & & & & & \\
\hline & Total & 134 & 3,47 & 0,72 & & & & & & \\
\hline \multirow{8}{*}{$\begin{array}{l}\text { Attitudes Towards Additive Containing } \\
\text { Nutrition }\end{array}$} & Body Building & 40 & 3.43 & 0.73 & Among & 1.946 & 5 & 0.389 & \multirow{3}{*}{0.75} & \multirow{3}{*}{0.59} \\
\hline & & & & & Groups & & & & & \\
\hline & Losing Weight & 10 & 3.73 & 0.86 & Within Group & 66.588 & 128 & 0.520 & & \\
\hline & Gaining Weight & 3 & 3.44 & 0.67 & Total & 68.534 & 133 & & & \\
\hline & Health & 29 & 3.28 & 0.63 & & & & & & \\
\hline & Conditioning & 30 & 3.28 & 0.68 & & & & & & \\
\hline & Fitness & 22 & 3.40 & 0.80 & & & & & & \\
\hline & Total & 134 & 3.38 & 0.72 & & & & & & \\
\hline \multirow{8}{*}{ Attitudes Towards Food Supplements } & Body Building & 40 & 2.59 & 1.06 & Among & 6.619 & 5 & 1.324 & \multirow{3}{*}{1.34} & \multirow{3}{*}{0.25} \\
\hline & & & & & Groups & & & & & \\
\hline & Losing Weight & 10 & 2.48 & 1.13 & Within Group & 126.448 & 128 & 0.988 & & \\
\hline & Gaining Weight & 3 & 2.42 & 1.38 & Total & 133.068 & 133 & & & \\
\hline & Health & 29 & 2.00 & 1.01 & & & & & & \\
\hline & Conditioning & 30 & 2.52 & 0.92 & & & & & & \\
\hline & Fitness & 22 & 2.43 & 0.81 & & & & & & \\
\hline & Total & 134 & 2.41 & 1.00 & & & & & & \\
\hline
\end{tabular}

Table 16. Values of relation in between participants' number of meals variable and AGN. AACN. AFS. BACN and BFS propositions

\begin{tabular}{cccc}
\hline & \multicolumn{3}{c}{ Number of meals } \\
\cline { 2 - 4 } & $\mathbf{n}$ & $\mathbf{r}$ & $\mathbf{p}$ \\
\hline $\begin{array}{c}\text { General Nutrition Attitudes } \\
\text { Attitudes Towards Additive } \\
\quad \text { Containing Nutrition }\end{array}$ & 180 & 0.08 & 0.32 \\
$\quad \begin{array}{c}\text { Attitudes Towards Food } \\
\quad \text { Supplements }\end{array}$ & 180 & 0.05 & 0.51 \\
$\begin{array}{c}\text { Behaviors Towards Additive } \\
\text { Containing Nutrition }\end{array}$ & 180 & $0.18^{*}$ & 0.02 \\
$\begin{array}{c}\text { Behaviors Towards Food } \\
\text { Supplements }\end{array}$ & 180 & 0.04 & 0.61 \\
\hline
\end{tabular}

When Table 15 is examined. no significant difference had been found among the participants' reason of attendance to gym variable and Propositions Regarding Attitudes Towards General Nutrition. Proposition Regarding Attitudes Towards Additive Containing Nutrition. Propositions Regarding Attitudes Towards Food Supplements. Propositions Regarding Behavior Towards Additive Containing 
Nutrition and Propositions Regarding Behaviors Towards Food Supplements.

When Table 16 is examined. while a significant relation in positive direction had been determined in between participants' number of meals variable and Propositions Regarding Behaviors Towards Additive Containing Nutrition ( $\mathrm{r}=0.18$; $\mathrm{p}=0.02$ ). no

Table 17. Results of one-way analysis of variance (ANOVA) in between participants' meal skipping variable and AGN. AACN. AFS. BACN and BFS

\begin{tabular}{|c|c|c|c|c|c|c|c|c|c|c|}
\hline & Group & $\mathbf{N}$ & $\bar{x}$ & ss & Var. Coeff. & K. T & sd & K. O & F & $p$ \\
\hline \multirow{7}{*}{$\begin{array}{l}\text { General } \\
\text { Nutrition } \\
\text { Attitudes }\end{array}$} & I don't skip & 45 & 3.73 & 0.55 & Among & 0.447 & 4 & 0.112 & \multirow{3}{*}{0.50} & \multirow{3}{*}{0.74} \\
\hline & & & & & Groups & & & & & \\
\hline & I do skip & 71 & 3.63 & 0.50 & Within Group & 39.063 & 174 & 0.224 & & \\
\hline & Breakfast & 30 & 3.60 & 0.38 & Total & 39.510 & 178 & & & \\
\hline & Lunch & 31 & 3.65 & 0.38 & & & & & & \\
\hline & Dinner & 2 & 3.85 & 0.12 & & & & & & \\
\hline & Total & 179 & 3.66 & 0.47 & & & & & & \\
\hline \multirow{7}{*}{$\begin{array}{l}\text { Attitudes } \\
\text { Towards } \\
\text { Additive } \\
\text { Containing } \\
\text { Nutrition }\end{array}$} & I don't skip & 45 & 2.30 & 1.02 & Among & 2.89 & 4 & 0.721 & \multirow{3}{*}{0.93} & \multirow{3}{*}{0.45} \\
\hline & & & & & Groups & & & & & \\
\hline & I do skip & 71 & 2.32 & 0.93 & Within Group & 135.536 & 174 & 0.779 & & \\
\hline & Breakfast & 30 & 2.07 & 0.66 & Total & 138.421 & 178 & & & \\
\hline & Lunch & 31 & 2.02 & 0.75 & & & & & & \\
\hline & Dinner & 2 & 2.14 & 0.20 & & & & & & \\
\hline & Total & 179 & 2.22 & 0.88 & & & & & & \\
\hline \multirow{7}{*}{$\begin{array}{l}\text { Attitudes } \\
\text { Towards Food } \\
\text { Supplements }\end{array}$} & I don't skip & 45 & 3.52 & 0.83 & Among & 0.767 & 4 & 0.192 & \multirow{3}{*}{0.37} & \multirow{3}{*}{0.83} \\
\hline & & & & & Groups & & & & & \\
\hline & I do skip & 71 & 3.44 & 0.71 & Within Group & 90.310 & 174 & 0.519 & & \\
\hline & Breakfast & 30 & 3.33 & 0.61 & Total & 91.077 & 178 & & & \\
\hline & Lunch & 31 & 3.37 & 0.64 & & & & & & \\
\hline & Dinner & 2 & 3.60 & 0.85 & & & & & & \\
\hline & Total & 179 & 3.43 & 0.72 & & & & & & \\
\hline \multirow{7}{*}{$\begin{array}{l}\text { Behaviors } \\
\text { Towards } \\
\text { Additive } \\
\text { Containing } \\
\text { Nutrition }\end{array}$} & I don't skip & 45 & 3.44 & 0.80 & Among & 2.586 & 4 & 0.647 & \multirow{3}{*}{1.08} & \multirow{3}{*}{0.37} \\
\hline & & & & & Groups & & & & & \\
\hline & I do skip & 71 & 3.23 & 0.88 & Within Group & 104.150 & 174 & 0.599 & & \\
\hline & Breakfast & 30 & 3.53 & 0.64 & Total & 106.736 & 178 & & & \\
\hline & Lunch & 31 & 3.43 & 0.59 & & & & & & \\
\hline & Dinner & 2 & 3.50 & 0.00 & & & & & & \\
\hline & Total & 179 & 3.37 & 0.77 & & & & & & \\
\hline \multirow{7}{*}{$\begin{array}{l}\text { Behaviors } \\
\text { Towards Food } \\
\text { Supplements }\end{array}$} & I don't skip & 45 & 2.40 & 1.15 & Among & 3.335 & 4 & 0.834 & \multirow{3}{*}{0.79} & \multirow{3}{*}{0.53} \\
\hline & & & & & Groups & & & & & \\
\hline & I do skip & 71 & 2.45 & 1.05 & Within Group & 183.128 & 174 & 1.052 & & \\
\hline & Breakfast & 30 & 2.23 & 0.79 & Total & 186.463 & 178 & & & \\
\hline & Lunch & 31 & 2.15 & 0.97 & & & & & & \\
\hline & Dinner & 2 & 3.00 & 1.06 & & & & & & \\
\hline & Total & 179 & 2.35 & 1.02 & & & & & & \\
\hline
\end{tabular}

When Table 17 is examined. no significant difference had been found among the participants' meal skipping variable and Propositions Regarding Attitudes Towards General Nutrition. Proposition Regarding Attitudes Towards Additive Containing Nutrition. Propositions Regarding Attitudes Towards Food Supplements. Propositions Regarding Behavior Towards Additive Containing Nutrition and Propositions Regarding Behaviors Towards Food Supplements. significant relation had been found among other propositions. 
Table 18. Results of one-way analysis of variance (ANOVA) in between participants' reason of meal skipping variable and AGN. AACN. AFS. BACN and BFS

\begin{tabular}{|c|c|c|c|c|c|c|c|c|c|c|}
\hline & Group & $\mathbf{N}$ & $\bar{x}$ & ss & Var. Coeff. & K. T & sd & K. O & F & $\mathrm{p}$ \\
\hline \multirow{6}{*}{$\begin{array}{l}\text { General } \\
\text { Attitudes }\end{array}$} & Losing Weight & 7 & 3.67 & 0.35 & Among Groups & 1.568 & 7 & 0.224 & \multirow{2}{*}{1.15} & \multirow{2}{*}{0.34} \\
\hline & Time & 68 & 3.63 & 0.38 & Within Group & 24.527 & 126 & 0.195 & & \\
\hline & Economy & 12 & 3.64 & 0.62 & Total & 26.094 & 133 & & & \\
\hline & Appetite & 31 & 3.71 & 0.46 & & & & & & \\
\hline \multirow[t]{3}{*}{ Nutrition } & Choosy Eating & 6 & 3.26 & 0.62 & & & & & & \\
\hline & Other & 6 & 3.54 & 0.52 & & & & & & \\
\hline & Fitness & 2 & 4.06 & 0.50 & & & & & & \\
\hline & Eating Out & 2 & 3.38 & 0.12 & & & & & & \\
\hline & Total & 134 & 3.63 & 0.44 & & & & & & \\
\hline \multirow{8}{*}{$\begin{array}{l}\text { Attitudes } \\
\text { Additive } \\
\text { Nutrition }\end{array}$} & Losing Weight & 7 & 1.57 & 0.51 & Among Groups & 3.883 & 7 & 0.555 & \multirow{2}{*}{0.79} & \multirow{2}{*}{0.60} \\
\hline & Time & 68 & 2.21 & 0.85 & Within Group & 88.761 & 126 & 0.704 & & \\
\hline & Economy & 12 & 2.38 & 0.74 & Total & 92.644 & 133 & & & \\
\hline $\begin{array}{r}\text { Towards } \\
\text { Containing }\end{array}$ & Appetite & 31 & 2.13 & 0.89 & & & & & & \\
\hline & Choosy Eating & 6 & 2.33 & 0.43 & & & & & & \\
\hline & Other & 6 & 2.38 & 1.18 & & & & & & \\
\hline & Fitness & 2 & 2.57 & 0.61 & & & & & & \\
\hline & Eating Out & 2 & 2.21 & 0.71 & & & & & & \\
\hline & Total & 134 & 2.19 & 0.83 & & & & & & \\
\hline \multirow{9}{*}{$\begin{array}{l}\text { Attitudes Towards } \\
\text { Food Supplements }\end{array}$} & Losing Weight & 7 & 3.23 & 0.94 & Among Groups & 5.186 & 7 & 0.741 & \multirow[b]{2}{*}{1.70} & \multirow{2}{*}{0.12} \\
\hline & Time & 68 & 3.35 & 0.57 & Within Group & 55.014 & 126 & 0.437 & & \\
\hline & Economy & 12 & 3.52 & 0.89 & Total & 60.200 & 133 & & & \\
\hline & Appetite & 31 & 3.63 & 0.69 & & & & & & \\
\hline & Choosy Eating & 6 & 3.00 & 0.77 & & & & & & \\
\hline & Other & 6 & 3.27 & 0.73 & & & & & & \\
\hline & Fitness & 2 & 4.00 & 0.85 & & & & & & \\
\hline & Eating Out & 2 & 2.60 & 0.28 & & & & & & \\
\hline & Total & 134 & 3.40 & 0.67 & & & & & & \\
\hline \multirow{2}{*}{$\begin{array}{r}\text { Towards } \\
\text { Containing }\end{array}$} & Losing Weight & 7 & 3.55 & 0.78 & Among Groups & 4.516 & 7 & 0.645 & \multirow{2}{*}{1.10} & \multirow{2}{*}{0.37} \\
\hline \multirow{7}{*}{$\begin{array}{l}\text { Behaviors } \\
\text { Additive } \\
\text { Nutrition }\end{array}$} & Time & 68 & 3.39 & 0.66 & Within Group & 73.987 & 126 & 0.587 & & \\
\hline & Economy & 12 & 3.15 & 1.02 & Total & 78.503 & 133 & & & \\
\hline & Appetite & 31 & 3.34 & 0.82 & & & & & & \\
\hline & Choosy Eating & 6 & 2.83 & 0.84 & & & & & & \\
\hline & Other & 6 & 3.64 & 1.09 & & & & & & \\
\hline & Fitness & 2 & 3.92 & 0.12 & & & & & & \\
\hline & Eating Out & 2 & 2.67 & 0.47 & & & & & & \\
\hline & Total & 134 & 3.35 & 0.77 & & & & & & \\
\hline \multirow{9}{*}{$\begin{array}{l}\text { Behaviors Towards } \\
\text { Food Supplements }\end{array}$} & Losing Weight & 7 & 2.32 & 1.10 & Among Groups & 4.232 & 7 & 0.605 & \multirow{2}{*}{0.62} & \multirow{2}{*}{0.74} \\
\hline & Time & 68 & 2.24 & 0.93 & Within Group & 123.550 & 126 & 0.981 & & \\
\hline & Economy & 12 & 2.52 & 1.06 & Total & 127.782 & 133 & & & \\
\hline & Appetite & 31 & 2.35 & 1.06 & & & & & & \\
\hline & Choosy Eating & 6 & 2.71 & 1.33 & & & & & & \\
\hline & Other & 6 & 2.25 & 0.81 & & & & & & \\
\hline & Fitness & 2 & 2.88 & 0.18 & & & & & & \\
\hline & Eating Out & 2 & 3.25 & 1.06 & & & & & & \\
\hline & Total & 134 & 2.34 & 0.98 & & & & & & \\
\hline
\end{tabular}

When Table 18 is examined. no significant difference had been found among the participants' reason of meal skipping variable and Propositions Regarding Attitudes Towards General Nutrition. Proposition Regarding Attitudes Towards Additive Containing Nutrition. Propositions Regarding Attitudes Towards Food Supplements. Propositions Regarding Behavior Towards Additive Containing
Nutrition and Propositions Regarding Behaviors Towards Food Supplements.

\section{CONCLUSION \& EVALUATION}

While a significant relation in positive direction had been determined in between age variable and Propositions Regarding Attitudes Towards General Nutrition. no significant relation had been found among other propositions. When the relevant 
literature is reviewed. it has been determined that in Öztürk's (2017) study. the age group of 35 and over is the group that uses the highest food supplement.

While significant difference had been determined in between gender and Propositions Regarding Behaviors Towards Food Supplements. no significant difference had been found among other propositions. In the Propositions Regarding Behaviors Towards Food Supplements. it had been determined that the averages are high in favor of males. When the studies performed on the subject are examined. the study performed by Öztürk (2017) had found a statistical significant difference in between male and female groups. It had been determined that this difference is in attitudes towards food supplements and that the male score higher compared to female in terms of attitudes developed towards food supplements in a statistically significant manner. in other words that they develop a more positive perception towards the food supplements. This condition has revealed the result that it may be associated with the tendency of males for using more supplements such as protein powder and lecarnetin etc. in body building. Roininen et al. (2000). Kaya et al. (2005). Demirezen and Coşansu (2005). Stafleu et al. (1994). and Lennernas et al. (1997) had found by their studies that females develop more negative attitude towards additive containing nutrients compared to males.

While a significant relation in positive direction had been determined among the variables of height and weight and Propositions Regarding Behaviors Towards Food Supplements. no significant relation had been found among other propositions.

While a significant difference had been determined in the variable of department and in Propositions Regarding Attitudes Towards General Nutrition. no significant difference had been found in other propositions. In the Propositions Regarding Attitudes Towards General Nutrition. it had been determined that it is in favor of the students of Department of Coaching from among the students of Department of Coaching and students of Department of Sports Management.

While a significant relation in positive direction had been determined in between the variable of weekly attendance to gym and Propositions Regarding Behaviors Towards Additive Containing Nutrition. no significant relation had been found among the other propositions.
While a significant relation in positive direction had been determined in between the variable of number of meals and Propositions Regarding Behaviors Towards Additive Containing Nutrition. no significant relation had been found among the other propositions. Önder et al. (2000). in their study performed with the students of final grade of high school. had determined that only $60.7 \%$ of the students are regularly having breakfast. and that the females skip the breakfast more frequently compared to males. Alphan et al. (2002) had determined in their study that $41.9 \%$ of the adolescents are skipping breakfast. $18.9 \%$ of them are skipping lunch. and $6.6 \%$ of them are skipping dinner. Y1lmaz and Öskan (2007) had determined in their research that the university students are frequency skipping meals. that the meal skipped the most is lunch. and the reason of skipping meal is primarily lack of time. and that the students are being nourished in an unbalanced manner. Aksoydan and Çakır (2011). by their study in which the nutritional habits. physical activity levels and BMI of adolescents were determined. had determined that the adolescents are skipping the breakfast the most (16.3\%). and that frequency of skipping of meal is more among secondary school students and females. In the study group. the frequency of ones having regular physical activity is very low. It had been determined that the state of being physically active is decreasing along with age. The ones in primary school are more active than the ones in secondary school. Males are performing more physical activity compared to females. Baş et al. (2005) had also found by their study that inactivity level in adolescent females is higher compared to males (37.6\% and $29.6 \%$ respectively). In the study of Öztora (2005). it had been determined only $15.7 \%$ of the adolescents are doing exercise for 4 hours and more per week.

As a result Karabulut and Altun (2018) significant correlations were found in the "Health Responsibility" and "Physical Activity" subscales of the HLSB scale. It was determined that scores of the females who regularly do sports are higher in both subdimensions $(p<0.05)$. The scores of the females who do sports regularly were found to be higher in the "Nutrition" subscale. No significant differences were determined in the subdimensions of "Spirituality". "Interpersonal Communication" and "Stress Management". and between the educational status and ages of females. As a result. doing sports regularly positively affects the responsibility of 
doing sports and physical activity level and - even though it is at low level- nutrition behavior. Education and age variables have no effect on healthy life style behaviors.

\section{REFERENCES}

1. Aksoydan E. Çakır N. Adölesanların beslenme alışkanlıkları. fiziksel aktivite düzeyleri ve vücut kitle indekslerinin değerlendirilmesi. Gülhane Tip Derg. 2011:64-270.

2. Akbaba N. Büyüme ve gelişmede beslenmenin önemi. Bilim Teknik Dergisi. 1991:24(280); 42-43.

3. Alphan ME. Keskin Y. Tatlı F. Özel okul ve devlet okulunda ögrrenim gören adölesan dönemindeki çocukların beslenme alışkanlıklarının karşılaştırılması. Beslenme ve Diyet Dergisi. 2002:31: 9-17. 11.

4. Aypek N. Kavak Ş. Ünüvar Ş. Çalışkan A. Gıda/Besin teknolojisi. Nobel Akademik Yayıncilık 2007.

5. Baş M. Altan T. Dinçer D. Aran E. Kaya HG. Yüksek O. Determination of dietary habits as a risk factor of cardiovascular heart disease in Turkish adolescents. Eur J Nutr. 2005:44: 174-182.

6. Büyüköztürk. Ş. (2012). Sosyal bilimler için veri analizi el kitabı. Ankara: Pegem A Yayıncilı.

7. Demirezen E. Coşansu G. Adölesan çağı öğrencilerde beslenme alışkanlıklarının değerlendirilmesi. STED 2005:14(8); 174-178.

8. Ersoy G. Okul çağı ve spor yapan çocukların beslenmesi. Anakara: Turna Ajans 2001.

9. Güneş Z. Spor ve beslenme - Antrenör ve sporcu el kitabı. Nobel Yayınevi. 2016.

10. Karabulut EO. Altun M. Düzenli olarak spor yapan ve yapmayan kadınların sağlıklı yaşam biçimi davranışlarının farklı değişkenler bakımından incelenmesi: Kırşehir ili örneği. KEFAD.2018:19(1); 936-948.

11. Kaya E. Sezek F. Köse EÖ. Sağllklı beslenmenin önemi ile ilgili öğrenci görüşlerinin cinsiyet. anne-baba eğitimi ve gelir düzeyi açısından incelenmesi. XIV. Ulusal Eğitim Bilimleri Kongresi. Pamukkale Üniversitesi Eğitim Fakültesi Yayınları. 2005:926-929.

12. Lennernas M. Fjellström C. Becker W. Grachettı I. Schmıtt A Remaut-De Winter AME. Kearney MJ. Influences on food choice perceived to be important by nationally-representative samples of adults in the European Union. European Journal of Clinical Nutrition. 1997:51(8); 8-15.

13. Merdol TK. Temel beslenme ve diyetetik. Güneş Tip Kitabevi 2015.

14. Önder FO. Kurdoğlu M. Oğuz G. Özben B. Atilla S. Oral SN. Gülveren Lisesi son sınıf öğrencilerinin bazı beslenme alışkanlıklarının saptanması ve bunun malnitrisyon prevelansı ile olan ilişkisi. Hacettepe Toplum Hekimliği Bülteni. http://www.thb. hacettepe.edu.tr/2000/20001.shtml. 2000.

15. Öztora S. İlköğretim çağındaki çocuklarda obezite prevalansının belirlenmesi ve risk faktörlerinin araştırılması. Yayınlanmamış Uzmanlık tezi. Dr. Sami Hatipoğlu Çocuk Sağlığı ve Hastalıkları Kliniği. İstanbul 2005.
16. Öztürk T. Spor salonlarına giden kişilerin genel beslenme alışkanlıkları. katkılı besinler ve gıda takviyeleri hakkındaki bilgi düzeyleri. görüş ve tutumları. Yüksek Lisans Tezi. İstanbul Gelişim Üniversitesi Sağlık Bilimleri Enstitüsü. İstanbul 2017.

17. Roınınen K. Lahteenmakı L. Tuorılla Hely. An application of means-end chain approach to consumers' orientation to health and hedonic characteristics of foods. Ecology of Food and Nutrition. 2000:39(1); 61-81.

18. Sezek F. Kaya E. Doğan S. Üniversite öğrencilerinin genel beslenme alışkanlıkları. katkılı besinler hakkındaki bilgi. görüş ve tutumları. Journal of Arts and Sciences. 2008:10. 117-134.

19. Stafleu A. Graaf CD. Van Staveren WA. De Jong MA. Attitudes Towards High-Fat Foods And Their Low-Fat Alternatives: Reliability and relationship with fat intake. Appetite. 22(1); 1994:183-196.

20. Yılmaz E. Özkan S. Üniversite öğrencilerinin beslenme alışkanlıklarının incelenmesi. Fırat Sağlık Hizmetleri Dergisi. 2007:2(6); 87-104. 